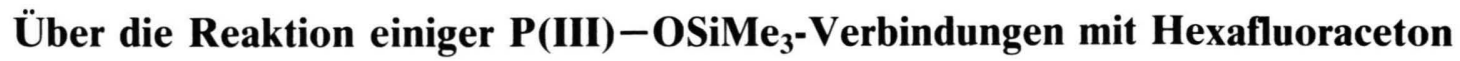

\author{
Reaction of Some P(III)- $\mathrm{OSiMe}_{3}$ Compounds with Hexafluoroacetone \\ Norbert Weferling und Reinhard Schmutzler* \\ Institut für Anorganische und Analytische Chemie der Technischen Universität, \\ Hagenring 30, 3300 Braunschweig \\ Z. Naturforsch. 43b, 1524-1528 (1988); eingegangen am 14. Juni 1988 \\ P(III)trimethylsilyl Phosphites, Hexafluoroacetone, \\ Bis(trimethylsilyl)perfluoropinacolyl Phosphoranes, \\ Perfluoropinacolyl Phosphonates, Pseudorotation Processes
}

The $\mathrm{P}(\mathrm{III})$ trimethylsilylesters, $\mathrm{RP}\left(\mathrm{OSiMe}_{3}\right)_{2}(\mathrm{R}=\mathrm{Me}, \mathrm{Ph})$ and $\mathrm{MeP}\left(\mathrm{OBu}^{n}\right)\left(\mathrm{OSiMe}_{3}\right)$, were synthesized by the silylation of the appropriate $\mathrm{P}(\mathrm{III})(: \mathrm{O})(\mathrm{H})$ compound with dimethylaminotrimethylsilane. Their reaction with hexafluoroacetone was studied with a view of synthesizing P-functional $\lambda^{5} \mathrm{P}$-trimethylsiloxy phosphoranes. From $\mathrm{RP}\left(\mathrm{OSiMe}_{3}\right)_{2}$ the expected bis(trimethylsilyl)perfluoropinacolyl phosphoranes, $\mathrm{RP}\left(\mathrm{OSiMe}_{3}\right)_{2}(\mathrm{pfp}=$ perfluoropinacolyl $)$, were obtained. Their stability was limited, and transformation to the known $\lambda^{4} \mathrm{P}$ species, $\mathrm{RP}(: \mathrm{O})(\mathrm{pfp})$, was observed. From the mixed $\mathrm{P}(\mathrm{III})$ ester, $\mathrm{MeP}\left(\mathrm{OBu}^{n}\right)\left(\mathrm{OSiMe}_{3}\right)$, formation of a $\lambda^{5} \mathrm{P}$ species was not observed, and the product was the same, $\mathrm{MeP}(: \mathrm{O})(\mathrm{pfp})$. A possible isomerization scheme for perfluoropinacolylbis(trimethylsiloxy)phosphoranes is discussed.

Die Umsetzung des Trimethylsilylphosphinits, $\mathrm{Me}_{2} \mathrm{POSiMe}_{3}$ (1) mit Hexafluoraceton (HFA) (2) liefert in hoher Ausbeute das Siloxyphosphoran (3) (Gl. (1)) [1 a, 1 b]:

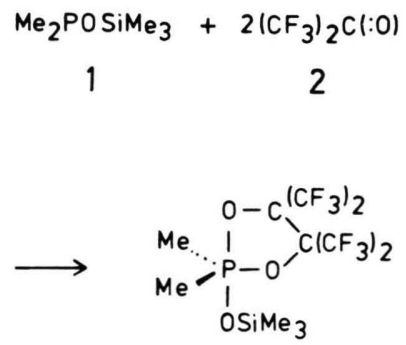

3
(1)

Auf Grund der Reaktivität der $\lambda^{5} \mathrm{P}-\mathrm{O}-\mathrm{SiMe}_{3}-$ Gruppe ist 3 weiteren Umsetzungen zugänglich und dient als Vorstufe für die Synthese neuartiger Verbindungen mit dem $\mathrm{Me}_{2} \mathrm{P}(\mathrm{pfp})$-System (pfp = Perfluorpinakolyl, $\left.\mathrm{OC}\left(\mathrm{CF}_{3}\right)_{2} \mathrm{C}\left(\mathrm{CF}_{3}\right)_{2} \mathrm{O}\right)$ [1].

Es war daher von Interesse, oxidative AdditionsReaktionen des HFA entsprechend Gl. (1) auch auf andere $\mathrm{P}(\mathrm{III}) \mathrm{OSiMe}_{3}$-Verbindungen auszudehnen; z.B. auf solche mit mehr als einer $\mathrm{OSiMe}_{3}$-Gruppe am Phosphoratom, wie $\mathrm{RP}\left(\mathrm{OSiMe}_{3}\right)_{2}(\mathrm{R}=\mathrm{Me}, \mathrm{Ph})$ 5a und 5b, oder auf P(III)-Verbindungen mit je einer Alkylester- und einer $\mathrm{Me}_{3} \mathrm{SiO}-G r u p p e$ am

\footnotetext{
* Sonderdruckanforderungen an Prof. Dr. R. Schmutzler. Verlag der Zeitschrift für Naturforschung, D-7400 Tübingen $0932-0776 / 88 / 1200-1524 / \$ 01.00 / 0$
}

Phosphor, wie 7. Die Reaktion von Tris(trimethylsilyl)phosphit, $\left(\mathrm{Me}_{3} \mathrm{SiO}\right)_{3} \mathrm{P}^{2}$ und der gemischten Ester, $\left(\mathrm{R}^{1} \mathrm{O}\right)\left(\mathrm{R}^{2} \mathrm{O}\right) \mathrm{POSiMe}_{3}\left(\mathrm{R}^{1}, \mathrm{R}^{2}=\right.$ Kohlenwasserstoffgruppen bzw. $\mathrm{R}^{1}=$ Kohlenwasserstoffgruppe; $\mathrm{R}^{2}=\mathrm{Me}_{3} \mathrm{Si}$ ) mit $\mathbf{2}$ ist kürzlich beschrieben worden [3]. Dabei wurden im Normalfall keine stabilen Verbindungen mit $\lambda^{5} \mathrm{POSiMe}_{3}$-Gruppen, sondern Abwandlungsprodukte mit tetra-koordiniertem $\left(\lambda^{4}\right)$ Phosphor erhalten .

Die Ausgangsverbindungen 5a, $5 \mathbf{b}$ bzw. 7 wurden durch Silylierung der Vorstufen, $\mathrm{RP}(\mathrm{H})(: \mathrm{O})(\mathrm{OH}) 4 \mathbf{a}$ und 4b bzw. $\mathrm{MeP}\left(\mathrm{OBu}^{\prime \prime}\right)(: \mathrm{O})(\mathrm{H}) \quad \mathbf{6}$ mittels $\mathrm{Me}_{2} \mathrm{NSiMe}_{3}$ in glatter Reaktion erhalten (Gl. (2) und (3)):

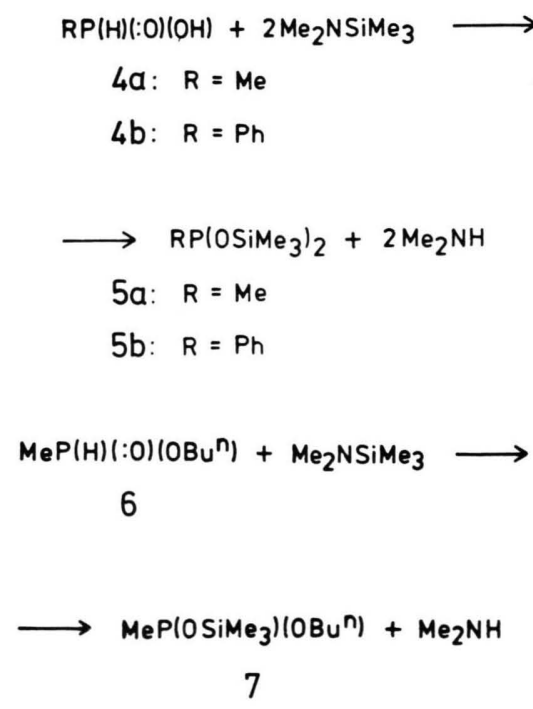

(3) 
Die verwendete Veresterungsmethode hat gegenüber anderen Verfahren [4] den Vorteil, daß die Aufarbeitung wesentlich einfacher ist. Für die Verbindung 4a wurde als Silylierungsreagenz auch die schwächere Base $\left(\mathrm{Me}_{3} \mathrm{Si}\right)_{2} \mathrm{NH}$ eingesetzt; dabei blieb die Reaktion auf der Stufe des Monoesters (8) stehen (Gl. (4)).

\section{$2 \mathrm{MeP}(\mathrm{H})(\mathrm{O})(\mathrm{OH})+\left(\mathrm{Me}_{3} \mathrm{Si}_{2} \mathrm{NH} \longrightarrow\right.$ \\ $4 a$ \\ $\longrightarrow 2 \mathrm{MeP}(\mathrm{H})(: \mathrm{O})\left(\mathrm{OSIMe}_{3}\right)+\mathrm{NH}_{3}$}

8

Diese Beobachtung verdient unter dem Gesichtspunkt der Schutzgruppenfunktion der Trimethylsiloxy-Gruppe eine gewisse Beachtung.

Umsetzung der Trimethylsilylphosphonite 5a, 5b und 7 mit $H F A, 2$

Es wurde erwartet, daß die oxidative Addition von $\mathbf{2}$ an die Verbindungen 5a, 5b und 7 interessante bifunktionelle Phosphorane liefern sollte. Im Falle des aus 7 resultierenden Produkts sollte die Reaktivität der beiden $\lambda^{5} \mathrm{P}$-ständigen Esterfunktionen stark unterschiedlich sein.

Es zeigte sich jedoch, daß das erwartete 1,3,2 $\lambda^{5}$ Dioxaphospholan 9 bei der Umsetzung von $7 \mathrm{mit}$ HFA nicht isoliert werden konnte. Als Produkt wurde vielmehr das bekannte 2-Methyl-1,3,2-dioxa4,4,5,5-tetrakis(trifluormethyl)-2-oxa- $\lambda^{4}$-phospholan (10) erhalten [5] (Kp., ${ }^{19} \mathrm{~F}-,{ }^{1} \mathrm{H}-,{ }^{31} \mathrm{P}-\mathrm{NMR}-$ Spektren) (Gl. (5)).
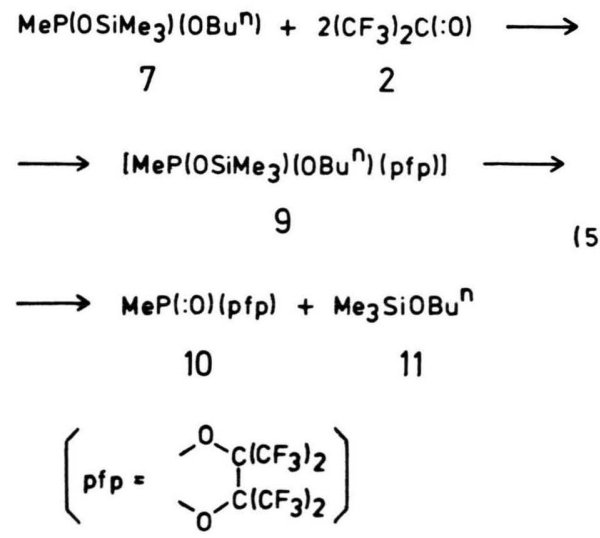

Bei der Umsetzung von 5a mit HFA wurden neben der erwarteten Verbindung 12, je nach Reaktionsführung, wechselnde Mengen von 10 erhalten (Gl. (6)).

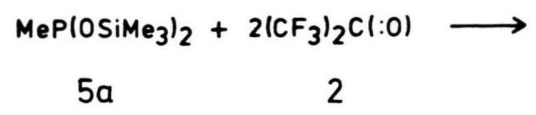

(6)

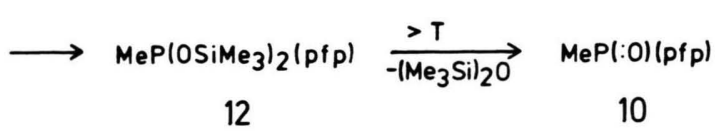

Die Ausbeute an $\mathbf{1 2}$ war besonders hoch, wenn das Reaktionsgemisch langsam von $-78^{\circ} \mathrm{C}$ auf Raumtemperatur erwärmt wurde. Eine destillative Reinigung von 12 war nicht möglich. Bei einem Druck von $0,01 \mathrm{~mm}$ und einer Ölbadtemperatur von $95^{\circ} \mathrm{C}$ zersetzte sich die Verbindung unter Bildung von $\mathbf{1 0}$ und Hexamethyldisiloxan (13), die destillativ getrennt und NMR-spektroskopisch identifiziert wurden.

Die Phenylverbindung $\mathbf{5 b}$ reagierte wie $5 \mathbf{a}$ mit zwei Äquivalenten HFA unter Bildung des 1,3,2 $\lambda^{5}$ Dioxaphospholans (14), einer öligen, farblosen Flüssigkeit (Gl. (7)):

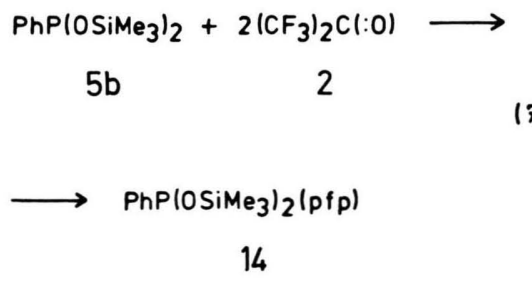

Das nach Gl. (7) erhaltene Rohprodukt 14 konnte durch Destillation ebenfalls nicht gereinigt werden. Ein Druckanstieg während der Vakuumdestillation des Reaktionsproduktes wies auf einen thermischen Zerfall von 14 hin. Das erwartete Thermolyseprodukt $\mathrm{PhP}(: \mathrm{O})(\mathrm{pfp})\left(\delta_{\mathrm{p}}+34,9 \mathrm{ppm}[6]\right)$ wurde jedoch überraschenderweise nicht gefunden. Die ${ }^{31} \mathrm{P}-\mathrm{NMR}$ Spektren zeigten vielmehr das Vorliegen mehrerer phosphorhaltiger Produkte an: niedriger siedende Fraktion (Kp. $86-95^{\circ} \mathrm{C} / 0,01 \mathrm{~mm} ; \delta_{\mathrm{p}}+2,3,+10,2$ und $+18,8 \mathrm{ppm})$; höhersiedende Fraktion (Kp. $118^{\circ} \mathrm{C} / 0,01 \mathrm{~mm} ; \delta_{\mathrm{p}}+2,3$ und $\left.+11,8 \mathrm{ppm}\right)$.

Bei einer unter besonders milden Bedingungen durchgeführten Umsetzung nach Gl. (7) wurden neben dem Hauptprodukt $14\left(\delta_{\mathrm{p}}+2,3 \mathrm{ppm}\right)$ ca. $5 \%$ Nebenprodukte $\left(\delta_{\mathrm{p}}+5,8,+10,2 \mathrm{ppm}\right)$ erhalten. 
Die ${ }^{19} \mathrm{~F}$-NMR-Daten (zwei Signalgruppen bei $\delta_{\mathrm{F}}-65,6$ und $-68,4 \mathrm{ppm}$; Intensitätsverhältnis 1:1) und das Massenspektrum (mit einem intensiven Molekülion) beweisen die Identität von 14.

\section{${ }^{19} \mathrm{~F}$-NMR-Untersuchungen an $\mathrm{RP}\left(\mathrm{OSiMe}_{3}\right)_{2}(\mathrm{pfp})$ $(R=M e: 12 ; R=P h: 14)$}

Im Hinblick auf die Möglichkeit von Pseudorotationsprozessen wurden die ${ }^{19} \mathrm{~F}$-NMR-Spektren der o. a. Verbindungen in Abhängigkeit von der Temperatur untersucht. Für Pseudorotationsprozesse an Phosphoranen des Typs $\mathrm{R}_{2} \mathrm{R}^{1} \mathrm{P}(\mathrm{pfp})$ läßt sich für $\mathrm{R}=$ $\mathrm{OSiMe}_{3}$ folgendes Isomerisierungsschema aufstellen.

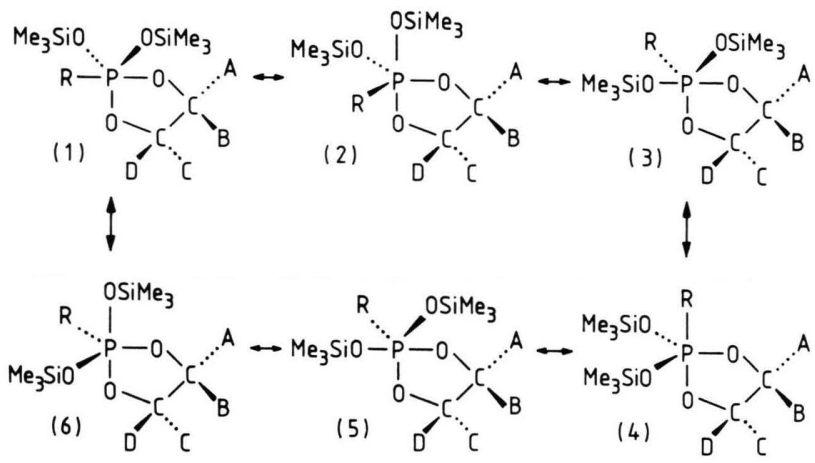

Fig. 1. Isomerisierungsschema für $4,4,5,5$-Tetrakis(trifluormethyl)-1,3,2 $\lambda^{5}$-dioxaphospholane des Typs $\mathrm{RP}\left(\mathrm{OSiMe}_{3}\right)_{2}(\mathrm{pfp}) ; \mathrm{A}=\mathrm{B}=\mathrm{C}=\mathrm{D}=\mathrm{CF}_{3}$.

Die Isomerisierungen $(2) \rightleftarrows(3)$ sowie $(5) \rightleftarrows(6)$ stellen sogenannte Niedrigenergieschritte der Umlagerung topomerer trigonal-bipyramidaler Konformationen gleicher Energie dar. Sie führen zur Äquilibrierung der $\mathrm{CF}_{3}$-Gruppen $\mathrm{A}$ und $\mathrm{C}$ sowie $\mathrm{B}$ und $\mathrm{D}$. Die Prozesse (1) $\rightleftarrows(2),(3) \rightleftarrows(4)$ sowie (6) $\rightleftarrows(1)$ stellen dagegen Hochenergieschritte dar, bei denen ein Ligand geringer Apicophilizität [9] in eine axiale Position überführt wird. Durch diesen Prozeß findet eine Äquilibrierung von A und B sowie C und D statt. Berechnungen $[10,11]$ zeigen, daß Pseudorotationsprozesse wie (2) $\rightleftarrows$ (3) nur eine geringe Aktivierungsenergie benötigen und daher selbst bei tiefen Temperaturen, relativ zur NMR-Zeitskala, rasch ablaufen.
In den ${ }^{19} \mathrm{~F}$-NMR-Spektren der Verbindungen $\mathbf{1 2}$ und 14 wurden bei Raumtemperatur zwei Signalgruppen $\left(\mathrm{R}=\mathrm{Me} ; \delta_{\mathrm{F}}=-65,9,-68,5 ; \mathrm{R}=\mathrm{Ph} ; \delta_{\mathrm{F}}=\right.$ $-65,8,-68,3)$ im Intensitätsverhältnis $1: 1$ beobachtet, die unterschiedliche Feinstruktur aufwiesen. Das bedeutet, daß der Hochenergieschritt bei Raumtemperatur „eingefroren“ war. Bis zur thermischen Zersetzung der beiden Verbindungen (kurzfristig wurde bis auf $160^{\circ} \mathrm{C}$ erhitzt) konnte keine Äquilibrierung der Isomere (z. B. (1) $\rightleftarrows(2))$ nachgewiesen werden Die nach folgender Näherungsgleichung [12] $\Delta \mathrm{G}^{*}=4,57 \mathrm{~T}_{\mathrm{c}}\left(9,97+\log \mathrm{T}_{\mathrm{c}} / \Delta v\right) \mathrm{cal} / \mathrm{mol}(8)$ $\left(\Delta \mathrm{G}^{*}=\right.$ Aktivierungsenthalpie; $\mathrm{T}_{\mathrm{c}}=$ Koaleszenztemperatur; $\Delta v=$ Abstand der Signale bei langsamem Austausch $(\mathrm{Hz})$ ) berechnete freie Aktivierungsenthalpie für diesen Prozeß ist somit höher als $21 \mathrm{kcal} / \mathrm{mol}$.

Die bisher durchgeführten Versuche zur Darstellung der bifunktionellen Phosphorane $\mathrm{RP}(\mathrm{pfp})\left(\mathrm{OSiMe}_{3}\right)_{2}(\mathrm{R}=\mathrm{Me}, \mathrm{Ph})$ haben die prinzipielle Darstellbarkeit dieser Verbindungen bewiesen. Wegen ihrer thermischen Instabilität, insbesondere aber wegen der schwierigen Reindarstellung, wurde von der weiteren Untersuchung dieser Substanzklasse abgesehen.

\section{Experimentelles}

Bei der Durchführung der Versuche wurden die bei der Handhabung von feuchtigkeits- und sauerstoffempfindlichen Substanzen üblichen Vorsichtsmaßnahmen beachtet.

\section{Ausgangsmaterialien}

Phosphonigsäuren 4a und 4b: Die Darstellung erfolgte in Anlehnung an Lit. [13].

Phosphonigsäure-bis(trimethylsilylester), 5a und $5 \mathbf{b}$ : Die Darstellung von 5a ist unten beschrieben, die von $\mathbf{5 b}$ erfolgte nach Lit. [14].

Methylphosphonigsäure- $n$-butylester 6 wurde von Dr. H. J. Kleiner (Hoechst AG) zur Verfügung gestellt. N, $\mathrm{N}^{\prime}$-Dimethylaminotrimethylsilan: Lit. [16].

NMR-Spektren: JEOL JNMC 60 HL Kernresonanzspektrometer; $60 \mathrm{MHz}\left({ }^{1} \mathrm{H}\right)$, Ref. $\mathrm{Si}\left(\mathrm{CH}_{3}\right)_{4}$; $56,4 \mathrm{MHz}\left({ }^{19} \mathrm{~F}\right)$, Ref. $\mathrm{CCl}_{3} \mathrm{~F} ; 24,3 \mathrm{MHz}\left({ }^{31} \mathrm{P}\right)$, Ref. $85 \%$ ext. $\mathrm{H}_{3} \mathrm{PO}_{4}$. Verschiebungen zu tieferem Feld, relativ zum angegebenen Standard, sind mit positivem, solche zu höherem Feld mit negativem Vorzeichen angegeben. - Massenspektren: AEI Instrument MS 9, $70 \mathrm{eV}$. E. I. 
Darstellung von Methylphosphonigsäure-bis(trimethylsilylester) (5a)

In einem 250-ml-Zweihalskolben mit Tropftrichter und Rückflußkühler wurden $7,7 \mathrm{~g}(0,097 \mathrm{~mol}) \mathbf{4 a}$ eingetragen und auf $75^{\circ} \mathrm{C}$ erhitzt. Dazu wurden $25 \mathrm{~g}$ $(0,214 \mathrm{~mol}) \mathrm{Me}_{2} \mathrm{NSiMe}_{3}$ getropft. Nach $16 \mathrm{~h}$ Rühren wurde überschüssiges $\mathrm{Me}_{2} \mathrm{NSiMe}_{3}$ bei R.T. $(20 \mathrm{~mm}$ ) abgezogen. Das verbleibende Produkt 5a wurde zweimal rektifiziert; Kp. $43{ }^{\circ} \mathrm{C}(9 \mathrm{~mm})$; Ausbeute $7,6 \mathrm{~g}(35 \%)$.

$\mathrm{C}_{7} \mathrm{H}_{21} \mathrm{O}_{2} \mathrm{PSi}_{2}(224,39)$

Gef. C 37,54 H 9,15,

Ber. C 37,47 H 9,43.

NMR-Daten: ${ }^{1} \mathrm{H}: \delta_{\mathrm{H}} 0,33\left(\mathrm{SiMe}_{3}\right), 1,23(\mathrm{PMe})$, ${ }^{2} J(\mathrm{HP}) 8,7$.

Umsetzung von Methylphosphonigsäure mit

Hexamethyldisilazan; Darstellung von

Methylphosphonigsäure-trimethylsilylester (8)

In einem 250-ml-Kolben mit Rückflußkühler wurde ein Gemisch von $14 \mathrm{~g}(0,175 \mathrm{~mol}) \mathbf{4 a}$ und $28,2 \mathrm{~g}$ $(0,115 \mathrm{~mol}) \mathrm{Hexamethyldisilazan} 16 \mathrm{~h}$ bei $90{ }^{\circ} \mathrm{C}$ gerührt. Dabei wurde der Monoester 8 in quantitativer Ausbeute erhalten.

${ }^{1} \mathrm{H}-\mathrm{NMR}-\mathrm{Daten}: \delta_{\mathrm{H}} 0,46\left(\mathrm{SiMe}_{3}\right), \delta_{\mathrm{H}} 1,63(\mathrm{DD}$, $\mathrm{PMe}),{ }^{2} J(\mathrm{HP}) 17,{ }^{3} J(\mathrm{HH}) 3, \delta_{\mathrm{H}} 7,4$ (DQ, HP), ${ }^{1} J(\mathrm{HP}) 540,{ }^{3} J(\mathrm{HH}) 3$.

Das in vorstehendem Experiment erhaltene Produkt 8 wurde mit $20,5 \mathrm{~g}(0,176 \mathrm{~mol}) \mathrm{Me}_{2} \mathrm{NSiMe}_{3}$ versetzt und $6 \mathrm{~h}$ bei $110^{\circ} \mathrm{C}$ am Rückfluß gekocht. Nach dem Abziehen der bei R.T./100 mm flüchtigen Anteile wurde das Rohprodukt fraktionierend destilliert; Kp. $45^{\circ} \mathrm{C}(10 \mathrm{~mm})$; Ausbeute an 5a $36,3 \mathrm{~g}$ $(92 \%)$.

NMR-Daten 5b: ${ }^{1} \mathrm{H}: \delta_{\mathrm{H}} 0,2\left(\mathrm{SiMe}_{3}\right), 7,4(\mathrm{Ph})$ breit; ${ }^{31} \mathrm{P}: \delta_{\mathrm{p}}+137,0$.

\section{Darstellung von Methylphosphonigsäure-trimethyl- silyl-n-butylester (7)}

Ein Gemisch von $6(11,8 \mathrm{~g}, 0,087 \mathrm{~mol})$ und $\mathrm{Me}_{2} \mathrm{NSiMe}_{3}(13,3 \mathrm{~g}, 0,114 \mathrm{~mol})$ wurde $16 \mathrm{~h}$ am Rückfluß gekocht. Nach dem Abziehen des überschüssigen Silylamins bei R.T./100 mm wurde das verbleibende Produkt i. Vak. destilliert; Kp. $47^{\circ} \mathrm{C}$ (5 mm); Ausbeute 14,7 g (81\%).

\section{$\mathrm{C}_{8} \mathrm{H}_{21} \mathrm{O}_{2}$ PSi $(208,31)$}

Gef. C 45,91 H 10,16,

Ber. C 46,13 H 10,16.

NMR-Daten: ${ }^{1} \mathrm{H}: \delta_{\mathrm{H}} 0,38\left(\mathrm{SiMe}_{3}\right), 1,25(\mathrm{PMe})$, ${ }^{2} J(\mathrm{HP}) \quad 8,2,2,2 \quad\left(\mathrm{OCH}_{2} \mathrm{CH}_{2} \underline{\mathrm{CH}}_{2} \underline{\mathrm{CH}}_{3}\right)$, breit, 3,8 $\left(\mathrm{OCH}_{2} \mathrm{CH}_{2} \mathrm{CH}_{2} \mathrm{CH}_{3}\right)$, breit; ${ }^{31} \mathrm{P}: \delta_{\mathrm{p}}+169,7$.
Umsetzung der Phosphonigsäure-bis-(trimethylsilylester) 5a, $5 \mathbf{b}$ und $\mathbf{7}$ mit Hexafluoraceton HFA; $\mathbf{2}$

Der Phosphonigsäureester wurde in ein dickwandiges Glasrohr eingefüllt, 2 ( $c a$. 2,2 Äquivalente) bei $-196^{\circ} \mathrm{C}$ aufkondensiert, und das Glasrohr abgeschmolzen. Die Reaktionsmischungen wurden innerhalb $2 \mathrm{~d}$ auf Raumtemperatur gebracht. Danach wurde erneut auf $-196^{\circ} \mathrm{C}$ abgekühlt, überschüssiges HFA abkondensiert und das Reaktionsgemisch ${ }^{31} \mathrm{P}$ NMR-spektroskopisch untersucht.

Ansatzgrößen

P(III)-OSiMe $3^{-} \quad$ HFA Ausbeute

Verbindung

5a $15,5 \mathrm{~g}(0,069 \mathrm{~mol}) \quad 26 \mathrm{~g}(0,157 \mathrm{~mol}) \quad 38 \mathrm{~g}(99 \%)$

5b $14,8 \mathrm{~g}(0,05 \mathrm{~mol}) \quad 18,5 \mathrm{~g}(0,111 \mathrm{~mol}) \quad 30,4 \mathrm{~g}(95 \%)$

$710,0 \mathrm{~g}(0,048 \mathrm{~mol}) \quad 19,2 \mathrm{~g}(0,115 \mathrm{~mol}) \quad 16,3 \mathrm{~g}(63 \%)$

Die Umsetzung von 5a mit HFA lieferte das analysenreine Phosphoran 12, während aus der analogen Umsetzung von $5 \mathrm{~b}$ die Verbindung 14 mit einer NMRspektroskopisch bestimmten Ausbeute von ca. $90 \%$ gebildet wurde (Nebenprodukte mit $\delta_{\mathrm{p}}+5,8$, $+10,5 \mathrm{ppm})$. Aus der Umsetzung von 7 mit HFA wurde das erwartete Phosphoran 9 nicht erhalten. Im Rohprodukt wurde die $\lambda^{4} \mathrm{P}$-Verbindung $\operatorname{MeP}(: O)(p f p), 10$ zu $75 \%$ nachgewiesen; ein Nebenprodukt mit $\delta_{\mathrm{p}}+24,8$ konnte nicht identifiziert werden. Wegen seiner thermischen Instabilität konnte das Phosphoran 14 nicht rein dargestellt werden.

MeP(:O)(pfp), 10: Kp. $47{ }^{\circ} \mathrm{C}(1 \mathrm{~mm})$; MS: $m / z 394$ $\left(\mathrm{M}^{+}\right)$. - NMR-Daten: ${ }^{1} \mathrm{H}: \delta_{\mathrm{H}} 2,48(\mathrm{D}),{ }^{2} J(\mathrm{HP}) 18,6$; ${ }^{19} \mathrm{~F}: \delta_{\mathrm{F}}-69,0 ;{ }^{31} \mathrm{P}: \delta_{\mathrm{p}}+48,8$.

$\mathrm{MeP}\left(\mathrm{OSiMe}_{3}\right)_{2}$ (pfp) 12: MS: $m / z 556\left(\mathrm{M}^{+}\right)$. NMR-Daten: ${ }^{1} \mathrm{H}: \delta_{\mathrm{H}} 0,57\left(\mathrm{SiMe}_{3}\right), 1,85(\mathrm{PMe}, \mathrm{D})$, ${ }^{2} J(\mathrm{HP}) 18,5 ;{ }^{19} \mathrm{~F}: \delta_{\mathrm{F}}-65,9(\mathrm{M}),-68,5(\mathrm{M}) ;{ }^{31} \mathrm{P}$ : $\delta_{\mathrm{p}}+12,9$.

$\mathrm{C}_{13} \mathrm{H}_{21} \mathrm{~F}_{12} \mathrm{O}_{4} \mathrm{PSi}_{2}(556,45)$

Gef. C 28,10 H 3,82 P 5,73,

Ber. C 28,06 H 3,82 P 5,73.

$\mathrm{PhP}\left(\mathrm{OSiMe}_{3}\right)_{2}$ (pfp), 15: MS: $m / z 618\left(\mathrm{M}^{+}\right)$. NMR-Daten: ${ }^{1} \mathrm{H}: \delta_{\mathrm{H}} 0,45\left(\mathrm{SiMe}_{3}\right), 7,8(\mathrm{Ph}$, breit $) ;{ }^{19} \mathrm{~F}$ : $\delta_{\mathrm{F}}-65,6(\mathrm{M}),-68,4(\mathrm{M}) ;{ }^{31} \mathrm{P}: \delta_{\mathrm{p}}+2,3$.

Dem Fonds der Chemischen Industrie, Frankfurt am Main, wird für finanzielle Unterstützung, Dr. H. J. Kleiner (Hoechst AG) und Dr. Y. Ohsaka, Daikin Kogyo Co., Ltd, Osaka/Japan für die großzügige Überlassung von Chemikalien, Herrn Dr. H. M. Schiebel für die Aufnahme der Massenspektren und Herrn Professor G.-V. Röschenthaler (Universität Bremen) für wertvolle Diskussionen gedankt. 
[1] a) M. Volkholz, Dissertation, Technische Universität Braunschweig (1976);

b) M. Volkholz, O. Stelzer und R. Schmutzler, Chem. Ber. 111, 890 (1978).

[2] R. Bohlen, R. Francke, J. Heine, R. Schmutzler und G. V. Röschenthaler, Z. Anorg. Allg. Chem. 533, 18 (1986).

[3] J. Heine und G. V. Röschenthaler, Z. Naturforsch. 43b, 196 (1988).

[4] K. Issleib und B. Walter, Angew. Chem. 79, 59 (1967).

[5] V. N. Volkovitskii, I. L. Knunyants und E. G. Bykhovskaya, Zhur. Vses. Khim. Obshch. 18, 114 (1973); C. A. 78, 159752 d (1973).

[6] G. V. Röschenthaler, Habilitationsschrift, Technische Universität Braunschweig (1981).

[7] S. Trippett und P. J. Whittle, J. Chem. Soc. Perkin I 1973, 2302.
[8] J. A. Gibson, G. V. Röschenthaler und R. Schmutzler, J. Chem. Soc. Dalton Trans. 1975, 918.

[9] S. Trippett, Phosphorus Sulfur 1, 89 (1976).

[10] S. A. Bone, S. Trippett, M. M. White und P. J. Whittle, Tetrahedron Lett. 1974, 1795.

[11] A. Rauk, L. C. Allen und K. Mislow, J. Am. Chem. Soc. 94, 3035 (1972).

[12] H. Günther, NMR-Spektroskopie, S. 248, Georg Thieme Verlag, Stuttgart (1973).

[13] G. M. Kosolapoff und J. S. Powell, J. Am. Chem. Soc. 72, 4291 (1950).

[14] E. P. Lebedev, A. N. Pudovik, B. N. Tsyganov, R. Ya. Nazmutdinov und G. U. Romanov, Zhur. Obsh. Khim. 47, 698 (1977).

[15] K. A. Petrov, N. K. Bliznyuk, Yu. N. Studnev und A. F. Kolomiets, Zhur. Obshch. Khim. 31, 179 (1961).

[16] M. J. C. Hewson und R. Schmutzler, Inorg. Synth. 18, 179 (1978). 